\title{
Pulse Tube Cryocooler: Phasor Analysis and One-Dimensional Numerical Simulation
}

\author{
N. Almtireen ${ }^{1}$ (D) . J. J. Brandner ${ }^{1}$ J. G. Korvink ${ }^{1}$
}

Received: 23 May 2019 / Accepted: 30 January 2020 / Published online: 13 February 2020

(c) The Author(s) 2020

\begin{abstract}
An analysis of the detailed operation for the tube element is proposed for an orifice pulse tube cryocooler. This is achieved through phasor analysis using basic thermodynamic relations to estimate the approximated cooling power associated with this machine. Moreover, the effect of the phase shift angle is illustrated by forming an analogy between the phase shift mechanism and a series RLC circuit model. Next, a one-dimensional model based on the conservation equations of mass and energy is presented; the reduced model is solved numerically, for the temperature and velocity of the gas along the tube, to determine the mass flow and time-averaged enthalpy flows at the cold and hot ends of the tube. The findings from the one-dimensional analysis are compared with the phasor analysis results and validated by comparison with similar studies in the literature.
\end{abstract}

Keywords Orifice pulse tube cooler · Phasor analysis · 1D numerical simulation · Temperature dynamics

\section{Introduction}

In recent years, many advances have been reported in cryogenics, particularly for cryocoolers, that have led to an expansion of their potential applications, and even to new application areas, such as in superconducting devices [1, 2], IR sensors [3-5], compact magnetic resonance magnets [6], and various medical and space applications [7-9]. Among the cryocoolers reported, pulse tube coolers (PTCs) are of special interest. They are widely used due to their excellent features, including low weight, high efficiency, excellent reliability, compactness, and relatively short response time. Unlike other regenerative devices, like Stirling and Gifford-McMahon (G-M) cryocoolers, PTCs lack mechanical moving components (i.e. mechanical

\footnotetext{
J. G. Korvink

jan.korvink@kit.edu

1 Institute of Microstructure Technology, Karlsruhe Institute of Technology, 76344 Eggenstein-Leopoldshafen, Germany
} 
displacers, or rubbing seals). Particularly at the cold end of the cooler, this results in a low vibration process, making PTCs a good choice for miniaturized cooling systems. Recuperative devices like Joule-Thomson cryocoolers (J-T) feature the major advantages of rapid cooling, the absence of any moving components, and thus also provide the potential to be miniaturized. However, their drawbacks, such as the possibility of valve clogging, their intrinsic inefficiency, relatively high charge pressure, and very short cooling duration, make them less favoured or even impossible to be used in many important applications [10].

Lowering the temperature at one end of a hollow tube with alternating pressure at the other end was first presented by Gifford and Longsworth [11], who referred to it as the basic pulse tube cryocooler (BPTC). Unfortunately, soon after, the concept was abandoned due to its inefficiency and the lack of clear interpretation for its principle of operation. In particular, the absence of any associated phase shift mechanisms made it hard to compete with other types of cryocoolers available at the time. Introducing phase shift components improved the PTC's efficiency and enhanced its performance to become equivalent to or even better than other cryocoolers. Several improvements have been made since Mikulin et al. [12] implemented the first orifice-type PTC, which was already capable of reaching $70 \mathrm{~K}$. In 1999, Xu et al. [13] managed to reach below $2 \mathrm{~K}$ using a three-stage pulse tube refrigerator.

Further analytical and numerical analyses are required for the development of more effective pulse tube cryocoolers, to eventually replace other conventional types of cryocoolers in many application fields. Thermodynamic analytical models, such as phasor analysis, could provide insights into the physical phenomena that would help to grasp the principles more clearly. Numerical dimensional models are vital in providing more accurate understanding for the gas behaviour in the pulse tube, especially with the high nonlinearity of the conservation equations.

This paper now presents a numerical one-dimensional analysis based on solving the conservation of both mass and energy equations and then compares the results with the approximated phasor model after validating the results with reports in the literature.

\section{Theory}

\subsection{Principle of Operation}

Pulse tube cryocoolers rely on the theory of oscillatory compression and expansion of an ideal gas within a closed volume to achieve the desired refrigeration. PTCs, like other regenerative cryocoolers, utilize the compression/expansion of working gas to remove heat. The main merit over other types of cryocoolers, like Stirling and Gifford-McMahon machines, is that PTCs do not need a mechanical displacer or even a motorized valve mechanism to guarantee that a phase shift is set between the pressure signal, provided by the compressor, and the volumetric flow rate in the tube. Consequently, reliable phase shift mechanisms are essential for the refrigeration process of a PTC to be of any significance. 
Figure 1 shows a schematic diagram for a conventional pulse tube cryocooler. The main idea behind the operation can be summed up as follows: an oscillatory high pressure signal from the compressor will compress the gas near the hot end, which would result in the temperature to rise near it; thus, the hot end is connected to a heat exchanger. Because of the oscillatory response, the phase shift mechanism, and a uniform pressure gradient along the tube, the gas will effectively expand in the pulse tube only near the cold end, causing the temperature there to decrease. Cycle by cycle, a finite temperature gradient will be formed across the tube as a result of this periodic pressurization/depressurization action. Therefore, the PTC is a harmonically driven system that will eventually settle into a periodic steady state.

\subsection{Main Components}

Conventional PTCs are composed of a few key parts as depicted in Fig. 1. The compressor is conventionally either of reciprocating or dual opposed piston type, with linearly moving magnets, its main function being to deliver an alternating pressure via controlling the electrical drive on the magnets. The regenerator is the most vital part in any PTC, characterized by its efficiency. It is generally of porous nature, conventionally containing stacked mesh screens and/or spherical particles with high heat capacity and low thermal conductivity, with the function of receiving heat during the pressurization phase and delivering it back to the returning gas during depressurization. Clearly an ideal regenerator should not store thermal energy after one cycle.

The aftercooler (AC) is introduced to pre-cool the incoming gas from the compressor, reducing the temperature at the hot end ( $\mathrm{HHX}$ ) and causing the regenerator to supply low temperature gas more efficiently. Continuous water or air cooling is required at both the hot end heat exchanger (HHX) and the aftercooler to pick up any generated heat, and thus maintaining these positions at room temperature. The cold end (CHX) acts like the evaporator in a classic vapour-compression refrigeration cycle and is the position where a target object is to be mounted.

Along with the regenerator, the pulse tube is also a crucial element for correct PTC operation. Its main function is to reject heat, through enthalpy flow, from the

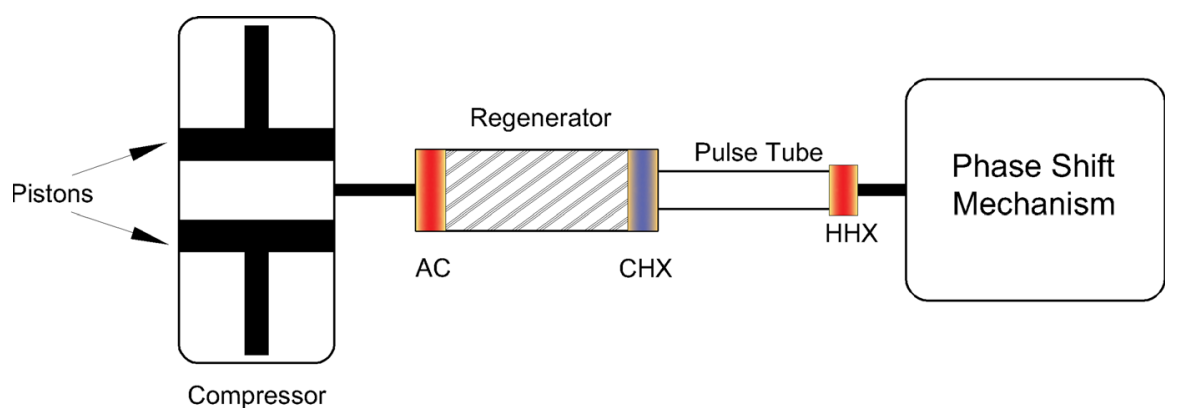

Fig. 1 Schematic diagram for conventional PTC, the hot end heat exchanger (HHX), the cold end heat exchanger (CHX), and the aftercooler (AC) is indicated (Color figure online) 
cold end to the hot end due to the presence of a phase shift mechanism. Conventionally, the pulse tube is a hollow tube made of stainless steel, with minimal thickness to reduce radial heat transfer, and optimized to provide a uniform temperature gradient along the tube between the cold and hot ends.

Phase shift mechanisms are essential to produce a significant cooling effect. The aim is to provide a phase shift, within the periodic cycle, between the source pressure signal and the resultant volumetric flow rate. The phase difference results in a steady temperature variation across the pulse tube. Conventionally, this has been achieved by introducing extra components including an orifice opening, an inertance tube, a buffer volume (reservoir), and/or a second bypass orifice after the hot end of the PTC system.

\subsection{Classifications}

In general, PTCs can be classified into sub-types according to their operation, their phase shift mechanism, and geometry. Table 1 lists different PTC types. In Stirlingtype coolers, unlike Gifford-McMahon types, the gas flow is not controlled via motorized valves, but rather by the alternating pressure signal itself, which controls the behaviour of the gas flow across the tube. Due to the switching mechanism, Gifford-McMahon types are considered to be low-frequency machines, where the efficiency of the system is adversely affected by the pressure signal being rectified using motorized controlled valves.

Inline PTCs are considered to be of simple geometry and have efficient operation since the gas flow is not changing direction, which would result in zero pressure drop. However, the drawbacks include low compactness and hard accessibility of the cold end, since it is located in the middle of the machine. U-type PTCs have advantages over the inline configuration, including easy-to-access cold end and size compactness. Unfortunately, U-type arrangements suffer from high pressure drop, deteriorating the efficiency of this configuration due to the $180^{\circ}$ degree change in flow direction. Generally, the pressure drop is dependent on the shape of the bend.

Coaxial and annular types share the same configuration with only one significant difference: whereas in the annular configuration the regenerator is inside the pulse tube, in the coaxial configuration, the assembly is reversed. These two types show the best compactness among all arrangements, the cold end being easily accessible with, like in the U-type configuration, the pressure drop being high. However, the pressure drop can be reduced using flow straighteners. Also, radial heat transfer between the regenerator and the pulse tube should be well characterized and minimized for this type to have proper operation.

Table 1 PTCs types and classifications

\begin{tabular}{lll}
\hline Operation & Geometry & Mechanism \\
\hline Gifford-McMahon (LF) & Inline & Orifice (OPTC) \\
Stirling (HF) & U-type & Double-inlet (DIPTC) \\
& Coaxial/annular & Inertance (IPTC) \\
\hline
\end{tabular}


Many PTCs with different phase shift mechanism designs are found in the literature that ensure the system achieves sufficient phase difference between the working pressure and flow rate, and to achieve acceptable temperature dynamics along the pulse tube. More details on these mechanisms, associated with an electrical analogy, are discussed in Sect. 3.1.3.

\section{Analysis}

The efficiency and compactness of PTCs have gained them significant importance for many applications. In particular, their potential should be utilized in microsystems applications, particularly due to their lack of moving components, and the absence of sealing or lubrication. However, in order to exploit the potential of a pulse tube cryocooler for miniaturization, it is imperative to relate its performance to the capabilities offered by a specific manufacturing technology. Normally, each application-technology combination permits only a small choice of materials, components, and operating conditions. For instance, replacing these components with more compatible versions, or modifying their designs for specific application, Huan et al. [14] presented a three-stage resonant cooling system replacing a conventional compressor with a thermo-acoustic engine to drive a pulse tube cryocooler. In their experiment with charge pressure of $3.5 \mathrm{MPa}$, the cold fingers reached a minimum temperature of $76 \mathrm{~K}$.

The efficient prediction of cooler operation in this context, which is necessary before embarking upon implementation, imposes the need for theoretical and experimental studies and to examine these studies for any inconsistency. Consequently, this paper aims to investigate the OPTC principle and evaluate the possibility to employ it later, either separately or in conjunction with other technologies, for microsystems and their applications. It studies the thermodynamic phenomena inside a miniature tube element of an OPTC first using a lumped system phasor analysis, and then by presenting a one-dimensional numerical analysis. It also describes an analogy between the PTC operation and RLC circuit analysis, which could help miniaturizing other components later.

\subsection{Phasor Analysis for OPTC}

Since phasor analysis was first introduced by Radebaugh [15], many authors have applied the method to different types of PTCs. Kittel et al. [16] used phasor analysis to show the fundamental thermodynamic behavior of a PTC. Hofmann and Pan [17] described the working principle of different types of PTCs with phasor diagrams. They suggested that these represent a simple possibility to find optimum phase shift angle. A phasor diagram for a DIPTC was presented by Chokhawala et al. [18]. Gardner and Swift [19] used those diagrams to introduce the inertance tube to an orifice type. They also studied the system using an electrical analogy and complex geometry approaches. This paper goes one step further and first analyzes the cooling power associated with 
standard OPTC machine using phasor analysis, to then compare it to the numerical analysis results in Sect. 3.2.1.

\subsubsection{Mass Flow Rate Analysis}

In this section, an orifice-type pulse tube cryocooler (OPTC) will be analyzed. The analysis assumes helium as the working fluid with time-variant pressure signal. Figure 2 shows the main components of an inline OPTC where the pressure source signal is sinusoidal and can be written as:

$$
P_{\mathrm{s}}[t]=p_{\mathrm{o}}+p_{1} \cos [2 \pi f t] .
$$

where the average pressure is $p_{o}$, pressure amplitude $p_{1}$, and the operating frequency $f$. The change in mass in the tube element is

$$
\mathrm{d} m_{\mathrm{pt}}=\mathrm{d} m_{\mathrm{h}}-\mathrm{d} m_{\mathrm{c}} .
$$

where $\mathrm{d} m_{\mathrm{h}}$ and $\mathrm{d} m_{\mathrm{c}}$ are the changes in mass near the hot and cold ends, respectively. For an orifice-type pulse tube cryocooler, the mass flow rate near the hot end is given by

$$
\dot{m}_{\mathrm{h}}[t]=\dot{m}_{\mathrm{or}}[t]=C_{\text {or }} \cdot \Delta p[t] .
$$

where $\dot{m}_{\text {or }}[t], C_{\text {or }}$, and $\Delta p$ are the mass flow rate near the orifice, the flow conductance of the orifice, and the pressure difference across the orifice, respectively. Assuming an adiabatic process with helium as monatomic ideal gas, the following equations are valid [16]:

$$
\begin{aligned}
p \cdot \mathrm{d} v & =R_{\mathrm{g}} T \cdot \mathrm{d} m ; \\
\left(\frac{T}{T_{\mathrm{o}}}\right) & =\left(\frac{p}{p_{\mathrm{o}}}\right)^{\left(\frac{\gamma-1}{\gamma}\right)} ; \\
p \cdot v_{\mathrm{pt}}^{\gamma} & =\text { constant; }
\end{aligned}
$$

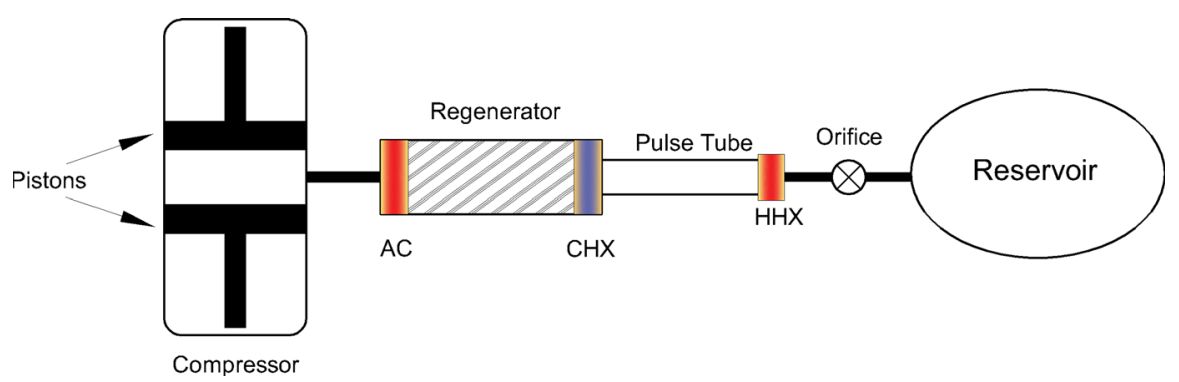

Fig. 2 Schematic diagram for conventional inline OPTC (Color figure online) 


$$
c_{\mathrm{p}}=\left(\frac{R_{\mathrm{g}} \gamma}{\gamma-1}\right) \text {. }
$$

where $p, \mathrm{~d} v, R_{\mathrm{g}}, T, \mathrm{~d} m, \gamma, v_{\mathrm{pt}}$, and $c_{\mathrm{p}}$ are the pressure, the volume change, the working gas constant, temperature, change in mass, the ratio of specific heat for helium, the pulse tube volume, and the constant pressure specific heat capacity of helium, respectively. $T_{\mathrm{o}}$ and $p_{\mathrm{o}}$ are the average temperature and pressure states. Differentiating Eq. (4c) will give $v_{\mathrm{pt}} \cdot \mathrm{d} p+\gamma p \cdot \mathrm{d} v_{\mathrm{pt}}=0$, and substituting it and Eq. (4a) in Eq. (2) leads to:

$$
\frac{\mathrm{d} m_{\mathrm{c}}[t]}{\mathrm{d} t}=\frac{v_{\mathrm{pt}}}{\gamma R_{\mathrm{g}} T_{\mathrm{c}}} \frac{\mathrm{d} p[t]}{\mathrm{d} t}+\frac{T_{\mathrm{h}}}{T_{\mathrm{c}}} \frac{\mathrm{d} m_{\mathrm{h}}[t]}{\mathrm{d} t} .
$$

where $T_{\mathrm{h}}$ and $T_{\mathrm{c}}$ are the temperature values at the hot and cold ends, respectively. Substituting Eq. (3), differentiating Eq. (1), and then substituting both results in Eq. (5) will give:

$$
\dot{m}_{\mathrm{c}}[t]=\frac{\omega p_{1} v_{\mathrm{pt}}}{\gamma R_{\mathrm{g}} T_{\mathrm{c}}} \cos \left[2 \pi f t+\frac{\pi}{2}\right]+\frac{T_{\mathrm{h}}}{T_{\mathrm{c}}} C_{\mathrm{or}} p_{1} \cos [2 \pi f t] .
$$

where $\omega$ is the angular frequency. Equation (6) shows that the gas mass flow rate at the cold end has a sinusoidal waveform that depends on the frequency and the pressure amplitude; $\dot{m}_{\mathrm{c}}[t]$ also depends on the dimension of the pulse tube and the cold end temperature. It should be noted that it is a resultant sum of two vectors, one of them being the mass flow rate at the hot end $\left(\dot{m}_{\mathrm{h}}[t]\right)$. Figure 3 shows the corresponding phasor diagram in which $\theta$ is the phase shift angle between the mass flow rate signal and the source pressure signal, i.e.

$$
\theta=\tan ^{-1}\left[\frac{\omega P_{1} v_{\mathrm{pt}}}{\gamma R_{\mathrm{g}} T_{\mathrm{c}}} / \frac{T_{\mathrm{h}} \dot{m}_{\mathrm{h}}}{T_{\mathrm{c}}}\right] .
$$

Fig. 3 The phasor diagram for the mass flow rates in the pulse tube. This shows that the mass flow rate near the cold end is a result of vector summation as illustrated in Eq. (6) (Color figure online)

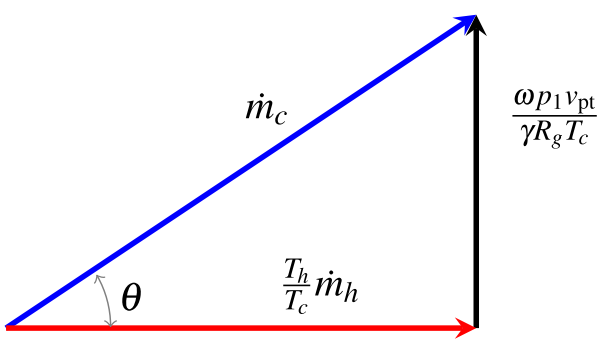




\subsubsection{Phase Shift Angle Effect on Cooling Power}

The previous section and Eq. (6) indicate that the mass flow rate at the cold end depends on many design parameters. Among these, the phase shift angle $\theta$ will be studied in this section. First, the enthalpy flow across the generator can be found using the first law of thermodynamics where the cooling power is:

$$
\dot{Q}_{\mathrm{c}}=\dot{H}_{\mathrm{pt}}-\dot{H}_{\mathrm{reg}},
$$

where $\dot{H}_{\mathrm{pt}}$ and $\dot{H}_{\text {reg }}$ are the enthalpy flow rates in the pulse tube and the regenerator, respectively. Assuming the regenerator is ideal, in a sense, it does not store any energy from one cycle to another. Then, $\dot{H}_{\text {reg }}=0$, which means for an ideal gas:

$$
\dot{Q}_{\mathrm{c}}=\dot{H}_{\mathrm{pt}}=\frac{c_{\mathrm{p}}}{\tau} \int_{0}^{\tau} \dot{m}_{\mathrm{c}}[t] T \cdot \mathrm{d} t .
$$

where $\tau$ is the pressure cycle period. Assuming the temperature is also a sinusoidal waveform, Eq. (4b) can be simplified using linear regression to (i.e. $\gamma \sim 1.67$ ):

$$
\left(\frac{T}{T_{\mathrm{o}}}\right)=0.4\left(\frac{p}{p_{\mathrm{o}}}\right) .
$$

Substituting Eq. (10) in Eq. (6), and then substituting both along with Eq. (4c) in Eq. (9) and cancelling out the integration of some periodic terms, will result in:

$$
\dot{Q}_{\mathrm{c}} \approx \frac{R_{\mathrm{g}} T_{\mathrm{c}} p_{1}}{2 p_{\mathrm{o}}}\left|\dot{m}_{\mathrm{c}}\right| \cos [\theta] .
$$

Equation (11) indicates that the cooling power is not only dependent on the pressure ratio between the amplitude and average pressure but also on the phase shift between the mass flow rate and the source pressure signal. As a result, the presence of $\theta$ in Eq. (11) implies that, theoretically, no significant cooling is done by a PTC unless there is some phase shift mechanism that ensures that $\theta$ is as small as possible where the maximum cooling power is reached when $\theta$ is zero. Additionally, Eq. (11) illustrates that the cooling power for a PTC drops as the temperature at the cold end drops, reducing the efficiency as predicted by the Carnot efficiency model.

\subsubsection{Phase Shift Mechanisms and RLC Circuit Analogy}

A number of publications present electrical analogy approaches which aim to interpret the phenomena of pulse tube dynamics, like in Refs. [19, 20]. Table 2 lists the key mechanical phenomena and their electrical analogies [21].

A harmonically driven series RLC circuit model is considered to be a good analogy for the purpose of studying the effect of different phase shift mechanisms, 
Table 2 RLC circuit analogy [21]

\begin{tabular}{lll}
\hline Quantity & Electrical analogy & Value \\
\hline Pressure & Voltage (V) & $P_{\mathrm{s}}$ \\
Flow rate & Current (I) & $Q=\left(\frac{\pi r^{4} \Delta P}{8 h \mu}\right)[22]$ \\
Compliance & Capacitance (C) & $C=\left(\frac{V}{\gamma p}\right)$ \\
Inertia of gas & Inductance (L) & $L=\left(\frac{\rho l}{A}\right)$ \\
Orifice resistance & Resistance (R) & $C_{\text {or }}$ \\
\hline
\end{tabular}

because it is also described by a second-order ordinary differential equation that is able to resonate. The resultant mechanical impedance $Z$, contributed to by all the phase shifting components, including the orifice, reservoir, and inertance tube, is associated with phase angle $\theta$. Both can be described by:

$$
\begin{gathered}
Z=R+j\left(\omega L-(\omega C)^{-1}\right) \\
\theta=\tan ^{-1}\left(\frac{\omega L-(\omega C)^{-1}}{R}\right) .
\end{gathered}
$$

where $Z$ and $\omega$ are the impedance and the angular frequency, respectively. The complex impedance goes to zero when $\omega=(\sqrt{L C})^{-1}$ or $\omega=\sqrt{A \gamma p} / \sqrt{\rho l V}$, which is the resonance condition. Equation (12b) shows that the inductance term will be significantly dominant in case of high-frequency machine and will be less dominant in case of low-frequency machine. This implies that an inertance tube is recommended with Stirling-type PTCs, while an orifice type or double-inlet are recommended for Gifford-McMahon machines. Also, in order to generate a phase difference in the tube, at minimum, an orifice associated with a reservoir is required. This explains why the original basic pulse tube cooler (BPTC), which is analogous to an electrical resistance circuit, failed to produce significant cooling; where in this particular case, the only cooling effect is caused due to the heat transfer from gas to the wall of the tube [23].

\subsection{One-Dimensional Numerical Analysis of OPTC}

\subsubsection{Mathematical Model}

Thermodynamic models, like in Refs. [24, 25], are important to understand the physics behind cooling action in PTCs, including phasor analyses which serve the purpose of explaining the PTC phenomena and giving insights on phase shift effect. Similarly, dimensional numerical models are required to have a clearer concept of the behaviour 
of the oscillating gas flow, the temperature dynamics, and the mass flow inside the pulse tube. These models are crucial due to the nonlinear-transient behaviour of the governing equations, which make them quite impossible to be solved analytically. Many forms for the governing equations of one-dimensional flow for compressed fluids can be found in the literature $[26,27]$. The mass, momentum, and energy conservation along with the equation of state can be written as [26]:

$$
\begin{gathered}
\frac{\partial \rho}{\partial t}+\frac{\partial(\rho u)}{\partial x}=0 \\
\rho\left(\frac{\partial u}{\partial t}+u \frac{\partial u}{\partial x}\right)=-\frac{\partial p}{\partial x}+\frac{4}{3} \frac{\partial}{\partial x}\left(\mu \frac{\partial u}{\partial x}\right) ; \\
\rho c_{p}\left(\frac{\partial T}{\partial t}+u \frac{\partial T}{\partial x}\right)=\frac{\partial p}{\partial t}+u \frac{\partial p}{\partial x}+\frac{\partial}{\partial x}\left(k_{\mathrm{g}} \frac{\partial T}{\partial x}\right) ; \\
p=\rho R_{\mathrm{g}} T .
\end{gathered}
$$

where $\rho, u, \mu$, and $k_{\mathrm{g}}$ are the gas density, the working gas velocity, the gas viscosity, and the gas thermal conductivity, respectively. These equations are built on the following assumptions:

- The working fluid is Newtonian, and helium is an ideal gas.

- The flow is laminar.

- Quasi-steady wall friction is only significant in two- or three-dimensional analysis.

- Viscous and inertial forces are not large enough to create substantial pressure change along the tube, hence resulting in uniform only time-dependent pressure along the tube (i.e. $\partial p / \partial x=0$ ).

Thus, the momentum Eq. (14) can be discarded (see [28, 29]). The pressure signal in Eq. (15) is sinusoidal and can be written as $p[t]=p_{o}+p_{1} \bar{p}[t]$, where $\bar{p}[t]$ is the varying part of the pressure signal. Substituting Eqs. (13) and (15), (16) can be rearranged after substituting $\rho T$ to obtain:

$$
\frac{\partial u}{\partial x}=\Phi \frac{\partial^{2} T}{\partial x^{2}}+f_{1}
$$

In the same manner, Eq. (15) can be reshaped to obtain the equation for the temperature:

$$
\frac{\partial T}{\partial t}=\Phi T \frac{\partial^{2} T}{\partial x^{2}}-u \frac{\partial T}{\partial x}+f_{2} T .
$$

Here, $\Phi, f_{1}$, and $f_{2}$ are defined by: 


$$
\begin{aligned}
f_{1} & =\left(\frac{1}{A_{o}+A_{1} \bar{p}[t]}\right)\left(\frac{A_{1}}{c_{p}}-A_{1}\right) \frac{\partial p}{\partial t} ; \\
f_{2} & =\left(\frac{1}{A_{o}+A_{1} \bar{p}[t]}\right)\left(\frac{1}{c_{p}}\right) \frac{\partial p}{\partial t} ; \\
\Phi & =\left(\frac{1}{A_{o}+A_{1} \bar{p}[t]}\right)\left(\frac{k_{\mathrm{g}}}{c_{p}}\right) ; \ni A_{o}=\left(\frac{p_{o}}{R_{\mathrm{g}}}\right), A_{1}=\left(\frac{p_{1}}{R_{\mathrm{g}}}\right) .
\end{aligned}
$$

Equation (18) is a nonlinear convection-diffusion partial differential equation (PDE). However, the diffusion term is attenuated by the diffusion coefficient $\Phi$, which was found to have a very small value, on the order of $\sim 10^{-8}$. This leads to a more dominant convective nature where the equation can be approximated to be of hyperbolic type.

A MATLAB® finite difference code was generated to solve the one-dimensional model numerically. First, the governing PDEs were discretized in both time and space after replacing all temperature and velocity derivatives with the finite difference equivalents, and finally, the numerical solution was computed using algorithm 1 (see Sect. 3.2.3).

\subsubsection{Initial and Boundary Conditions for the Pulse Tube}

Initial and boundary conditions are required to solve the system of equations. The tube is assumed to be one dimensional, with $0<x<l_{t}$, where $l_{t}$ is the length of the tube. The velocity of the gas at the hot end (i.e. $x=0$ ) needs to be defined as a boundary condition for Eq. (17) to be solved. This can be formulated by finding the volumetric flow of gas across the orifice and dividing it by the cross-sectional area of the tube to find the velocity at the hot end:

$$
u_{\mathrm{h}}[t]=-\frac{C_{\mathrm{or}}}{A_{t}}\left(\bar{p}[t]-\bar{p}_{r}[t]\right) .
$$

$\bar{p}[t]$ and $\bar{p}_{\mathrm{r}}[t]$ are the sinusoidal time pressure functions for the tube and the reservoir, respectively. According to the thermodynamic Poisson law, the pressure in the reservoir will encounter an adiabatic change with time due to the volumetric flow across the orifice. This can be expressed as:

$$
\dot{V}_{\mathrm{h}}[t]=\left(\frac{c_{\mathrm{p}} V_{\mathrm{r}}}{c_{\mathrm{v}} p_{\mathrm{r}}[t]}\right) \frac{\mathrm{d} p_{\mathrm{r}}[t]}{\mathrm{d} t} .
$$

where $V_{\mathrm{r}}$ and $c_{\mathrm{v}}$ are the reservoir volume and the constant volume specific heat capacity, respectively. Equation (21) is solved to find the change of pressure in the reservoir and thus finding the velocity of the gas in Eq. (20). Finally, two temperature boundary conditions at both the hot and cold ends are required for Eq. (18), which are: $T_{0}^{t}=T_{\mathrm{h}}$ and $T_{\mathrm{L}}^{t}=T_{\mathrm{c}}$. If the entire tube is considered to be at ambient temperature, one initial condition is needed for the temperature equation: $T_{x}^{0}=T_{\mathrm{h}}$. 


\subsubsection{Numerical Solution}

After the mathematical model is set, a numerical method is used to solve the differential equations Eqs. (17) and (18). First, two equally spaced computational time and space grids are introduced:

$$
\begin{aligned}
& x_{i}=i \lambda ; i=1, \ldots, N_{x} ; \Rightarrow \lambda=\frac{l_{t}}{N_{x}}, \\
& t^{k}=k \delta ; k=0, \ldots, N_{t} ; \Rightarrow \delta=\frac{1}{N_{t}} .
\end{aligned}
$$

The velocity and the temperature formulae are found after discretizing in both time and space using finite difference approximations. More specifically, a forward time centred space difference (FTCS) method, sometimes referred to as an explicit forward Euler method, was first adopted, as shown in Eqs. (22) and (23), to find the first- and second-order derivatives. These are:

$$
\begin{gathered}
u_{i}^{k}=u_{i-1}^{k}+\left(\frac{\Phi^{k}}{\lambda}\right)\left(T_{i-1}^{k}-2 T_{i}^{k}+T_{i+1}^{k}\right)+\lambda f_{1}^{k} ; \\
T_{i}^{k+1}=T_{i}^{k}+\delta\left(\frac{\Phi^{k} T_{i}^{k}}{\lambda^{2}}\right)\left(T_{i-1}^{k}-2 T_{i}^{k}+T_{i+1}^{k}\right)+\delta \Psi_{i}^{k}+\delta f_{2}^{k} T_{i}^{k} .
\end{gathered}
$$

where:

$$
\begin{aligned}
& \Psi_{N_{x}}^{k}=\left\{\begin{array}{l}
\left(\frac{-u_{i}^{k}}{\lambda}\right)\left(T_{i}^{k}-T_{i-1}^{k}\right) ; u_{i}^{k}>0 \\
\left(\frac{-u_{i}^{k}}{\lambda}\right)\left(T_{i+1}^{k}-T_{i}^{k}\right) ; u_{i}^{k}<0
\end{array} ;\right. \\
& \Psi_{i}^{k}=\left\{\begin{array}{l}
\left(\frac{-u_{i}^{k}}{2 \lambda}\right)\left(3 T_{i}^{k}-2 T_{i-1}^{k}+T_{i-2}^{k}\right) ; u_{i}^{k}>0 \\
\left(\frac{-u_{i}^{k}}{2 \lambda}\right)\left(3 T_{i+1}^{k}-2 T_{i}^{k}+T_{i-1}^{k}\right) ; u_{i}^{k}<0
\end{array} ;\right. \\
& u_{N_{x}}^{k}=u_{N_{x}-1}^{k}+\left(\frac{\Phi^{k}}{\lambda}\right)\left(T_{N_{x}}{ }^{k}-2 T_{N_{x-1}}^{k}+T_{N_{x-2}^{k}}\right)+\lambda f_{1}^{k} .
\end{aligned}
$$

The solution for the approximated temperature equation depends on the direction of the flow velocity. Hence, the function $\Psi$ determines which differencing scheme is used to solve this equation. Moreover, a first-order upwind/downwind model was used at the ends to avoid numerical noise and to prevent the occurrence of nonphysical oscillation, while a second-order upwind scheme was used for all inner elements. The generated code calculates the predicted temperature and velocity profiles for each time node $T_{i}^{k}$ and space node $u_{i}^{k}$. This code can be outlined as follows: 
- The code starts by calculating the pressure signals in the tube $\bar{p}[t]$ and the reservoir $\bar{p}_{\mathrm{r}}[t]$. It uses the previous values to evaluate the boundary condition for the velocity $u_{\mathrm{h}}$.

- Then, it computes the temperature $T_{i}^{k+1}$ depending on the $T_{i}^{k}$ and $u_{i-1}^{k}$ taking into consideration the direction of the flow (as illustrated by function $\Psi$ ) and the boundary conditions for the temperature at the hot and cold ends.

- It evaluates the velocity $u_{i}^{k}$ using the temperature value for the same time element. See Algorithm 1.

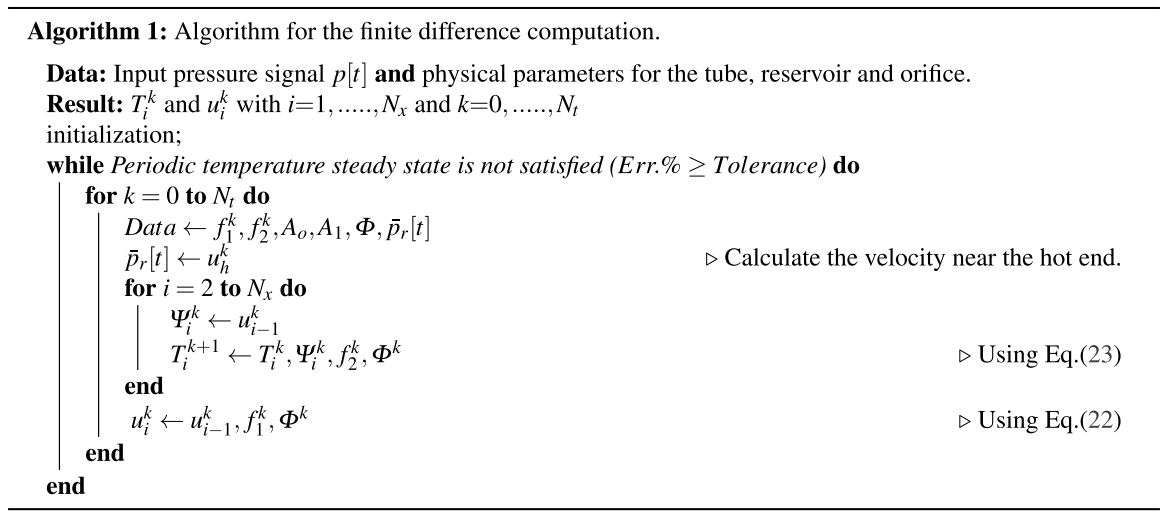

FTCS was first chosen due to its explicit approach. The values of the interior points are given by known data (i.e. boundary data or previously computed interior points). Unfortunately, the convergence of this algorithm is strongly sensitive to the value of $\left(\delta / \lambda^{2}\right)$, the ratio between time and space grids, which can noticeably affect its convergence if ill-chosen. Moreover, since increasing the operating frequency requires increasing the time grid points, this regime will fail when considering higher frequencies or even trying to study reduced tube lengths. However, it showed good results for systems with low operating frequency if a fine space grid is not vital. Thus, another algorithm was employed for higher operating frequency systems. The implicit BTCS (backward time centred space), sometimes referred to as the implicit backward Euler method, is a typical choice. This algorithm involves solving a system of instantaneous linear PDEs, which is expected to converge regardless of the size of time and space grids, because of its unconditional stability owing to the tridiagonal structure of the linear equation and the system matrix $A$ 's diagonal dominancy [30]. As a result, Eq. (23) is modified to:

$$
T_{i}^{k+1}=T_{i}^{k}+\delta\left(\frac{\Phi^{k} T_{i}^{k}}{\lambda^{2}}\right)\left(T_{i-1}^{k+1}-2 T_{i}^{k+1}+T_{i+1}^{k+1}\right)+\delta \Psi+\delta f_{2}{ }^{k+1} T_{i}^{k+1} .
$$


Equation (24) is then transformed to a system of linear equations that follow the general form $\left(A T_{i}^{k+1}=T_{i}^{k}+F\right)$ where $A$ and $F$ are matrices of constant elements at a given time instant. This can be expressed more explicitly as:

$$
\left[\begin{array}{cccc}
\Omega_{\mathrm{d}} & -\Omega_{2} & 0 & 0 \\
-\Omega_{1} & \ddots & \ddots & 0 \\
0 & \ddots & \ddots & -\Omega_{2} \\
0 & 0 & -\Omega_{1} & \Omega_{\mathrm{d}}
\end{array}\right] \cdot\left[\begin{array}{c}
T_{1}^{k+1} \\
T_{2}^{k+1} \\
\vdots \\
T_{N_{x}-1}^{k+1}
\end{array}\right]=\left[\begin{array}{c}
T_{1}^{k}+\Omega_{1} T_{0}^{k} \\
T_{2}^{k} \\
\vdots \\
T_{N_{x}-1}^{k}+\Omega_{2} T_{N_{x}}^{k+1}
\end{array}\right]
$$

Here, $\Omega_{1}, \Omega_{\mathrm{d}}$, and $\Omega_{2}$ are the elements in the $A$ and $F$ matrices whose values depend on the direction of the gas nodal velocity and are represented as:

$$
\begin{aligned}
\text { if } u_{i}^{k}<0 ; \Longrightarrow \Omega_{\mathrm{d}} & =1+\delta\left(\frac{2 \Phi^{k} T_{i}^{k}}{\lambda^{2}}\right)+\delta\left(f_{2}\right)^{k}-\left(\frac{\delta u_{i}^{k}}{\lambda}\right), \\
\Omega_{2} & =\left(\frac{\Phi^{k} T_{i}^{k}}{\lambda^{2}}\right), \Omega_{1}=\left(\frac{\Phi^{k} T_{i}^{k}}{\lambda^{2}}\right)-\left(\frac{\delta u_{i}^{k}}{\lambda}\right) . \\
\text { if, } u_{i}^{k}>0 ; \Longrightarrow \Omega_{\mathrm{d}} & =1+\delta\left(\frac{2 \Phi^{k} T_{i}^{k}}{\lambda^{2}}\right)+\delta\left(f_{2}\right)^{k}+\left(\frac{\delta u_{i}^{k}}{\lambda}\right), \\
\Omega_{2} & =\left(\frac{\Phi^{k} T_{i}^{k}}{\lambda^{2}}\right)+\left(\frac{\delta u_{i}^{k}}{\lambda}\right), \Omega_{1}=\left(\frac{\Phi^{k} T_{i}^{k}}{\lambda^{2}}\right) .
\end{aligned}
$$

In contrast to the previous algorithm, BTCS starts with computing the velocity for the current space/time element and then solves a system of linearized equations to find the corresponding space temperatures for each time step. Although the algorithm requires considerably higher computing effort compared to the explicit version, it acquires the merit to converge unconditionally to a solution. The quality of the computed solution, to some extent, depends on the size of the space element (i.e. the smaller the element, the better the results), as is expected from the finite difference method. The algorithm now allows to investigate the effect of different operating frequencies and reduced tube lengths.

\section{Results and Discussion}

\subsection{Application of the Model}

Figure 4 shows the tube, orifice, and the reservoir elements where the tube area, length, average pressure, and charging pressure were set to $10^{-3} \mathrm{~m}^{2}, 0.1 \mathrm{~m}, 3 \mathrm{MPa}$, and $0.3 \mathrm{MPa}$. The hot and cold end temperatures were set to $300 \mathrm{~K}$ and $70 \mathrm{~K}$, respectively, and the orifice flow conductance $C_{\text {or }}$ is set to $10^{-8} \mathrm{~m}^{3} \mathrm{~Pa}^{-1} \mathrm{~s}^{-1}$. 


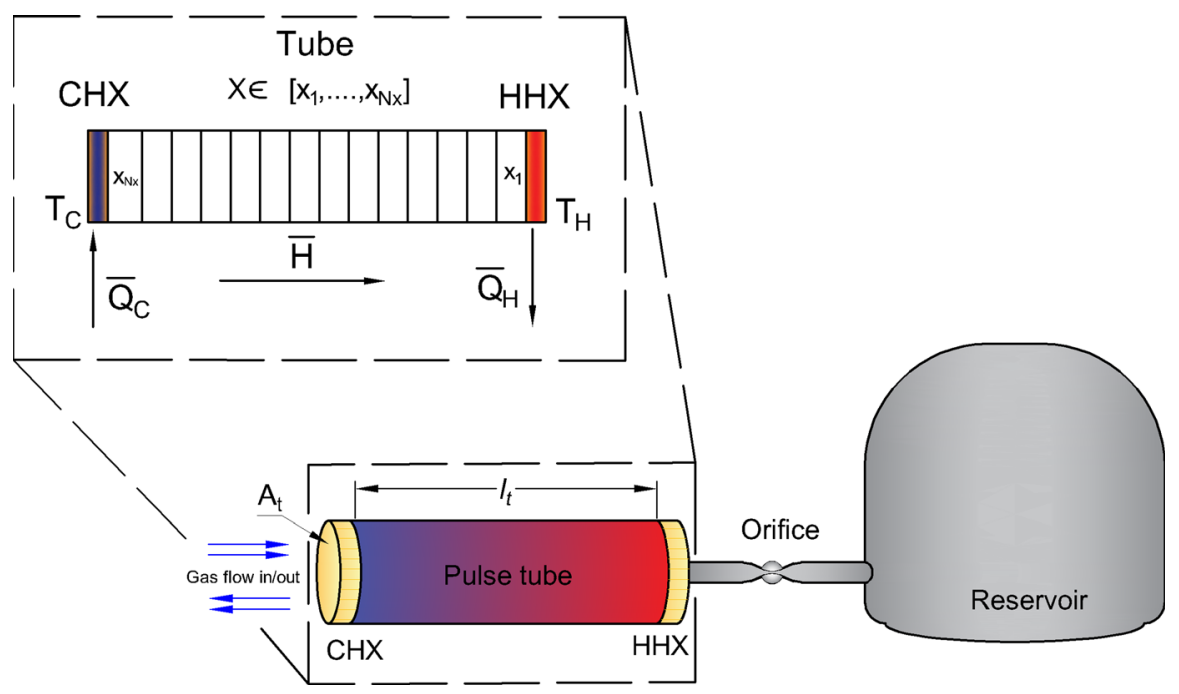

Fig. 4 Schematic diagram of the tube, orifice, and the reservoir elements with the tube physical and thermodynamic parameters along with the space grid shown in the zoomed-in diagram. HHX and CHX are kept at $300 \mathrm{~K}$ and $70 \mathrm{~K}$, respectively (Color figure online)

\subsection{Temperature Profiles}

The simulation starts with considering a low operating frequency, since the orifice type is conventionally considered to be a low-frequency machine. First, the simulation was run for a $10 \mathrm{~Hz}$ operating frequency for a tube of $10 \mathrm{~cm}$ in length. The temperature profiles varied with respect to the pressure cycle. As predicted previously, the code demonstrated that only periodic steady state can be reached. This state is reached after approximately ten cycles.

Figure 5 shows the steady-state temperature profiles converging to a solution for different corresponding locations on the pressure cycle with $10 \mathrm{~Hz}$ operating frequency. It clearly shows that the distribution of the temperature along the tube length is not uniform with respect to the pressure cycle, where intersections and overlapping between different profiles can be observed.

Figure 5 also illustrates the temperature profiles for $20 \mathrm{~Hz}$ operating frequency. The results exhibit better temperature profiles, where the profiles are more uniform across the tube, and the temperature is settling to the desired temperature near the cold end for all temperature profiles regardless of the corresponding location on the pressure cycle. After that, the model was run for higher operating frequencies reaching $80 \mathrm{~Hz}$ as depicted in Fig. 5. The results displayed nearly the same general shape for the temperature profiles as with $20 \mathrm{~Hz}$ operating frequency. However, it was noticed that the cold region was expanded and pushed further towards the hot region, which is, conventionally, not desired. Hence, the simulation results suggest that the optimum operating frequency for this system is near $20 \mathrm{~Hz}$. It is worth noting that the velocity at the cold end, and thus the mass flow rate, will rise with increasing frequency as predicted in Eq. (6), which would suggest that if a process 

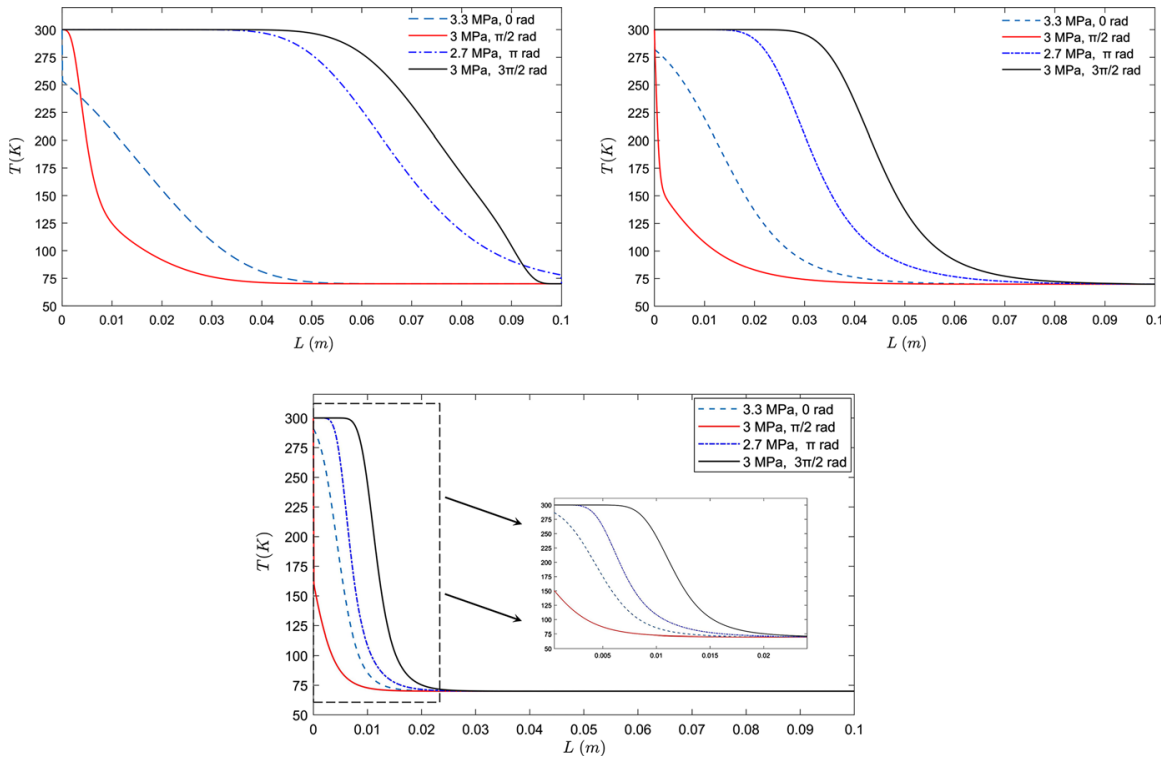

Fig. 5 Top left: Temperature profiles for OPTC with $10 \mathrm{~Hz}$. Top right: Temperature profiles for OPTC with $20 \mathrm{~Hz}$. Bottom: Temperature profiles for OPTC with $80 \mathrm{~Hz}$ (Color figure online)

with low vibrations is desired, a high-frequency system will have to be considered with caution.

\subsection{Enthalpy and Cooling Power}

If an ideal regenerator is assumed, the analytical values of cooling power, heat removed from the hot heat exchanger and thus enthalpy flow through the tube, are given by $[25,31]$ :

$$
\dot{Q}_{\mathrm{c}}=\dot{Q}_{\mathrm{h}}=\dot{H}_{\mathrm{pt}}=\frac{1}{2} \cdot C_{\mathrm{or}} \cdot p_{1}^{2} .
$$

Thus, the ideal enthalpy flow through the tube is found to be $450 \mathrm{~W}$. For the reasons mentioned in the previous section, an operating frequency of $20 \mathrm{~Hz}$ is further studied. Figure 6 illustrates the mass flow rate near both the cold and hot ends. Clearly, the mass flow rate at the cold end is higher than that at the hot end, since both the density and the velocity of the gas flow are much higher near the cold end.

Equation (9) relates the cooling power to the enthalpy flow, which in return depends on the mass flow rate near the cold end in case of an ideal regenerator. The enthalpy flows at the cold and hot ends are estimated by calculating the time-average value of the mass flow-temperature product at the cold and hot ends $(\sim 420 \mathrm{~W}$ and $434 \mathrm{~W}$, respectively, for $\lambda=5 \times 10^{-4}$ and $\delta=5 \times 10^{-4}$ ). The difference in these values is mostly due to discretization errors, the simplifications in physics (i.e. the momentum equation), and imperfect characterization of the orifice flow conductance or the pressure 
Fig. 6 Mass flow rate at the hot and cold ends (Color figure online)

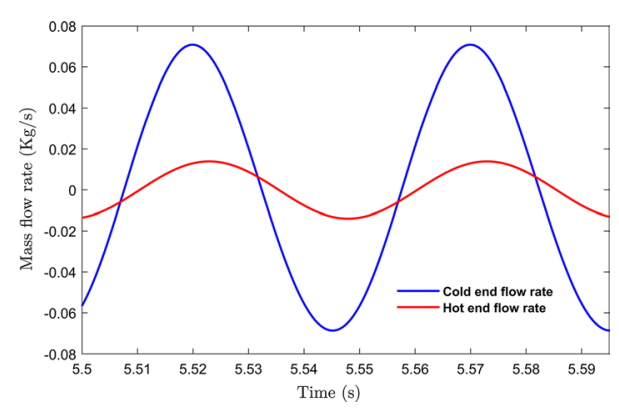

change in the reservoir due to the oscillatory motion of the gas in the tube. The approximated cooling power suggested by the phasor analysis in Eq. (11) is found to be around $\sim 470 \mathrm{~W}$ with phase shift angle of $0.3623 \mathrm{rad}$. The imbalance here is due to the fact that Eq. (11) is built on various assumptions and does not account for other losses and factors in the regenerator, reservoir, and other elements. Hence, it can be interpreted as best case scenario analysis. The analysis leads to the following conclusion: a cooling power of maximally $420 \mathrm{~W}$ can be reached using an OPTC with systems of similar specifications.

Table 3 lists key parameters for different PTCs with different physical specifications. In this study, the generated periodic temperature profiles can be compared to results found in the literature, particularly, the harmonic analysis in [24, 25] and the one-dimensional analysis in [29] where they all show similar time-dependent temperature profiles, in which the temperature dynamics can only predict periodic steady states. Generally, the average enthalpy, and hence the cooling power rating, is roughly determined by Eq. (25). The results for the estimated average enthalpy flows agree with some results obtained from the time-dependent axisymmetric CFD. In [32], the cold and hot ends' average enthalpy can be estimated to be $\sim 175 \mathrm{~W}$ and $225 \mathrm{~W}$, respectively, for two neighbouring operating frequencies of $15 \mathrm{~Hz}$ and $34 \mathrm{~Hz}$, but with different system specifications. Moreover, these time-averaged estimated enthalpy flows at the hot and cold ends agree with those estimated by the three-dimensional analysis in [31] for different system size. The different listed cooling power figures are due to different system specifications and simulation assumptions. For instance, many parameters can change the resulted net cooling power, such as porosity, the hydraulic diameter of the regenerator matrix, the thickness of the tubes, and the swept volume near the cold and the hot ends. Also the frequency, the average pressure, and the pressure ratio would alter the losses and affect the net cooling power. As a result, different incorporations of these parameters and other cryocooler components in the simulation study lead to different cooling power figures. 
Table 3 Different OPTC studies key parameters

\begin{tabular}{|c|c|c|c|c|}
\hline Reference & Study type & $\begin{array}{l}\text { Tube geometry (length } \\
\text { and area) }\end{array}$ & $\begin{array}{l}\text { Enthalpy flow/cooling } \\
\text { power/comments }\end{array}$ & $\begin{array}{l}\text { Boundary } \\
\text { conditions }\end{array}$ \\
\hline $\begin{array}{l}\text { De Waele } \\
\text { et al. }[24,25]\end{array}$ & $\begin{array}{l}\text { Thermody- } \\
\text { namics/ } \\
\text { harmonic } \\
\text { approx. }\end{array}$ & $0.2 \mathrm{~m}, 1 \times 10^{-4} \mathrm{~m}^{2}[24]$ & $\begin{array}{l}\text { General relationships } \\
\text { for: Cooling power and } \\
\text { temperature profiles. }\end{array}$ & $\begin{array}{l}T_{\mathrm{h}}=300 \mathrm{~K}, \\
T_{\mathrm{c}}=100 \mathrm{~K}\end{array}$ \\
\hline $\begin{array}{l}\text { Lyulina } \\
\text { et al. [29] }\end{array}$ & $1 \mathrm{D}$ & $0.2 \mathrm{~m}, 2 \times 10^{-2} \mathrm{~m}^{2}$ & $\sim 1258 \mathrm{~W}$ & $\begin{array}{c}T_{\mathrm{h}}=300 \mathrm{~K} \\
T_{\mathrm{c}}=70 \mathrm{~K}\end{array}$ \\
\hline $\begin{array}{l}\text { Antao and } \\
\text { Farouk [32] }\end{array}$ & $\begin{array}{l}\text { Axisymmetric } \\
\text { CFD }\end{array}$ & $0.06 \mathrm{~m}, 7.85 \times 10^{-5} \mathrm{~m}^{2}$ & $\sim 225 \mathrm{~W}(f=34 \mathrm{~Hz})$ & $\begin{array}{l}T_{\mathrm{h}}=293 \mathrm{~K}, \\
T_{\mathrm{c}}=100 \mathrm{~K}\end{array}$ \\
\hline $\begin{array}{l}\text { Willems and } \\
\text { Dam [31] }\end{array}$ & $3 \mathrm{D}$ & $0.2 \mathrm{~m}, 4.9 \times 10^{-4} \mathrm{~m}^{2}$ & $\begin{array}{l}1033 \mathrm{~W}(\text { ideal }) \\
675 \mathrm{~W} \text { (non-ideal) }\end{array}$ & $\begin{array}{c}T_{\mathrm{h}}=300 \mathrm{~K} \\
T_{\mathrm{c}}=70 \mathrm{~K}\end{array}$ \\
\hline Taylor et al. [33] & CFD & $<5 \times 10^{-4} \mathrm{~m}^{2}$ & $\sim 80 \mathrm{~W}$ (cooling power) & $\begin{array}{c}T_{\mathrm{h}}=300 \mathrm{~K} \\
T_{\mathrm{c}}=80 \mathrm{~K}\end{array}$ \\
\hline \multirow[t]{3}{*}{ This study } & Phasor & $0.1 \mathrm{~m}, 1 \times 10^{-3} \mathrm{~m}^{2}$ & $\sim 470 \mathrm{~W}$ & $\begin{array}{c}T_{\mathrm{h}}=300 \mathrm{~K} \\
T_{\mathrm{c}}=70 \mathrm{~K}\end{array}$ \\
\hline & $1 \mathrm{D}$ & $"$ & $\sim 420 \mathrm{~W}$ & \multirow{2}{*}{$\begin{array}{c}T_{\mathrm{h}}=300 \mathrm{~K} \\
T_{\mathrm{c}}=70 \mathrm{~K}\end{array}$} \\
\hline & Analytical & $"$ & $450 \mathrm{~W}$ & \\
\hline
\end{tabular}

\section{Conclusion}

In this paper, a general overview of the principle of operation for a pulse tube cryocooler (OPTC) was presented. After that, the effect of sinusoidal oscillating gas in the tube element of the OPTC was clarified first using phasor analysis associated with an electrical analogy to simplify the principle of phase shift mechanism, and second by numerically solving a one-dimensional model based on mass and energy conservation equations using an unconditionally stable implicit numerical algorithm. In the one-dimensional simulation model, the temperature settled to the desired temperature near the cold end and showed a condition of periodic steady state along a $10-\mathrm{cm}$ tube with uniform distribution and acceptable temperature gradient along the tube length for $20 \mathrm{~Hz}$ operating frequency. Furthermore, both mass and time-averaged enthalpy flows at the cold and hot ends were estimated; these findings were used to estimate the cooling power for this system, which can attain $\sim 420 \mathrm{~W}$ of cooling power. The results were first compared with that of the phasor thermodynamic analysis and secondly validated by similar studies in the literature for consistency. Future work will consider a two-dimensional model for a similar system in which the momentum conservation equation cannot be neglected, which should provide more accurate results and will allow to study more detailed phenomena. In conclusion, this study is a guide to estimate approximate design parameters for any similar cooling machine.

Acknowledgements Open Access funding provided by Projekt DEAL.

Open Access This article is licensed under a Creative Commons Attribution 4.0 International License, which permits use, sharing, adaptation, distribution and reproduction in any medium or format, as long as 
you give appropriate credit to the original author(s) and the source, provide a link to the Creative Commons licence, and indicate if changes were made. The images or other third party material in this article are included in the article's Creative Commons licence, unless indicated otherwise in a credit line to the material. If material is not included in the article's Creative Commons licence and your intended use is not permitted by statutory regulation or exceeds the permitted use, you will need to obtain permission directly from the copyright holder. To view a copy of this licence, visit http://creativecommons.org/licen ses/by/4.0/.

\section{References}

1. T. Ki, S. Jeong, Cryogenics 51(6), 341 (2011)

2. J.Y. Hu, E.C. Luo, L.M. Zhang, X.T. Wang, W. Dai, Appl. Energy 112, 1166 (2013)

3. A. Rogalski, Infrared Phys. Technol. 54(3), 136 (2011)

4. R. Radebaugh, in, Infrared Technology and Applications XXVI, vol. 4130 (2000), p. 17

5. R. Lewis, W. Yunda et al., Proc. SPIE 8012, 80122H-1 (2011)

6. O. Kirichek, P. Carr, C. Johnson, M. Atrey, Rev. Sci. Instrum. 76, 055104 (2005)

7. N. Rando, D. Lumb, M. Bavdaz, D. Martin, T. Peacock, Nucl. Instrum. Methods Phys. 522(1-2), 62 (2004)

8. R. Boyle, R. Ross Jr., AIP Conf. Proc. 613(1), 1037 (2002)

9. R. Radebaugh, Proc. IEEE 92(10), 1719 (2004)

10. Y. Zhao, G. Yu, J. Tan, X. Mao, J. Li, R. Zha, N. Li, H. Dang, Cryogenics 90, 30 (2018)

11. W.E. Gifford, R. Longsworth, J. Eng. Ind. 86(3), 264 (1964)

12. E. Mikulin, A. Tarasov, M. Shkrebyonock, Advances in Cryogenic Engineering (Springer, Berlin, 1984), pp. 629-637

13. M. Xu, A. De Waele, Y. Ju, Cryogenics 39(10), 865 (1999)

14. T. Huan, H. Jianying, Z. Limin, L. Ercang, Phys. Procedia 67, 445 (2015)

15. R. Radebaugh, Advances in Cryogenic Engineering (Springer, Belrin, 1990), pp. 1191-1205

16. P. Kittel, A. Kashani, J. Lee, P. Roach, Cryogenics 36(10), 849 (1996)

17. A. Hofmann, H. Pan, Cryogenics 39(6), 529 (1999)

18. M. Chokhawala, K. Desai, H. Naik, K. Narayankhedkar, Adv. Cryog. Eng. 45, 159 (2000)

19. D.L. Gardner, G.W. Swift, Cryogenics 37(2), 117 (1997)

20. A. Derick, C. Damu, T.K. Biju, IOP Conf. Ser. Mater. Sci. Eng. 278(1), 012154 (2017)

21. D.C. Karnopp, D.L. Margolis, R.C. Rosenberg, System Dynamics: Modeling, Simulation, and Control of Mechatronic Systems (Wiley, New York, 2012)

22. A. Scheidegger et al., The Physics of Flow Through Porous Media (University of Toronto Press, London, 1958)

23. L. Mohanta, M. Atrey, Cryocoolers 16, 299 (2011)

24. A. De Waele, P. Steijaert, J. Gijzen, Cryogenics 37(6), 313 (1997)

25. A. De Waele, P.P. Steijaert, J. Koning, Cryogenics 38(3), 329 (1998)

26. G.K. Batchelor, An Introduction to Fluid Dynamics (Cambridge Mathematical Library) (Cambridge University Press, Cambridge, 2000)

27. A.H. Shapiro, The Dynamics and Thermodynamics of Compressible Fluid Flow (Wiley, New York, 1953)

28. W. Smith, Cryogenics 41(8), 573 (2001)

29. I. Lyulina, R. Mattheij, A. Tijsseling, A.T. de Waele, Int. J. Nonlinear Sci. Numer. Simul. 5(1), 79 (2004)

30. W.Y. Yang, W. Cao, T.S. Chung, J. Morris, Applied Numerical Methods Using MATLAB (Wiley, New York, 2005)

31. D. Willems, J. Dam, AIP Conf. Proc. 613, 934-941 (2002)

32. D.S. Antao, B. Farouk, Cryogenics 51(4), 192 (2011)

33. R.P. Taylor, G. Nellis, S. Klein, AIP Conf. Proc. 985, 1445-1453 (2008)

Publisher's Note Springer Nature remains neutral with regard to jurisdictional claims in published maps and institutional affiliations. 